\title{
5.
}

\section{Free-to-Play or Pay-to-Win?}

Casual, Hardcore, and Hearthstone

Kenton Taylor Howard

Transactions of the Digital Games Research Association June 2019, Vol 4 No 3, pp 147-169

ISSN 2328-9422

(C) The text of this work is licensed under a Creative

Commons Attribution - NonCommercial -NonDerivative

4.0 License (http://creativecommons.org/licenses/by-nc-nd/ 2.5/).

IMAGES: All images appearing in this work are property of the respective copyright owners, and are not released into the Creative Commons. The respective owners reserve all rights.

\section{ABSTRACT}

"Casual" and "hardcore" are commonly used descriptive terms for games and gamers. While critics have discussed these terms with regards to game design and culture, "free-to-play" games like Blizzard's Hearthstone add a monetary dimension to such considerations. Players can play such games for free, but success at them often entails purchasing in-game content. These games are 
sometimes instead derisively referred to as "pay-to-win:” players who spend money win more often. Free-to-play games suggest that casual and hardcore depend on how much money a player spends on the game, in addition to measures like time investment or play practices. I argue that free-to-play games encourage casual players to become more hardcore by spending more money on them in addition to improving their skills at the game, using Hearthstone as a case study to examine the implications of the free-to-play pricing structure on both game design and game players.

\section{Keywords}

casual games, hardcore games, free-to-play games, collectible card games, Hearthstone

\section{INTRODUCTION}

In A Casual Revolution, Jesper Juul defined the qualities of what he calls "casual games," contrasting them with "hardcore" video games: while he noted that casual is a word with many potential meanings in terms of gaming, he argued that "simple casual games are more popular than hardcore games” (2010, 8). Since then, casual games have redefined how games are structured and how players play them, challenging the image of gaming as a hobby for hardcore enthusiasts. The differences between casual and hardcore games and gamers are common distinctions made within gaming culture, and Juul's description of casual games as being "simple" and "popular" mirrors the way such games are often described, suggesting that they are not particularly complex, that they have a broad appeal, and that they are aimed at a wider audience beyond the "hardcore gamer." Likewise, Chess claimed that "we can understand casual video games as those which are simple to learn and play, addictive enough that one can play them in short periods of time or for as long as time allows, and are cheap or free” (2013, 84). These descriptions of casual games recall the way the terms 
casual and hardcore are applied to players in gamer culture: they are status-based distinctions in which casual gamers are portrayed as less seriously dedicated to the hobby than hardcore players are. Casual games have changed since these texts were written, however, and free-to-play games like Blizzard Entertainment's Hearthstone (2014) have challenged the traits of casual games by adding hardcore elements to gameplay, raising questions about how such games, and those who play them, should be considered.

Critical approaches to the use of terms like casual and hardcore within gaming culture are often rhetorical, focusing on the implications of the terms. Many theorists have analyzed the rhetoric of games: for example, Paul argued that "if rhetorical analysis is a critical perspective, focusing beyond mere persuasion, all elements surrounding games are influential symbols worthy of study, as all games function persuasively" (2011). More specifically, theorists have looked at the players of games through the lens of terms like casual and hardcore: for example, Poels et al. claimed that "a clear categorization of the different types of game players is important for the academic world” (2011, 2). Culturally defined terms such as casual and hardcore are therefore worthy of analysis even if the rhetoric that underpins such terms is problematic.

Problems with the terms casual and hardcore usually arise in relationship to gender and gamer identity: for example, Soderman noted that the terms are frequently associated with gender, highlighting "the recent fears and anxieties expressed by the hardcore gamer community over the rise of casual games which can be linked to a distinctive gendering of the hardcore as masculine and the casual as feminine” (2009). Likewise, Eklund argued that "the casualization/feminization of gaming seems a pervasive ideology in digital games culture” $(2016,19)$, pointing out that discussions about casual games often focus on the way they challenge the idea that video games are for male players. In some cases, critics point out that casual games expand the idea of who video games are for in general: for example, Chiapello 
claimed that "[casual games] eclipse the video game stereotype of shooting games and the male teen player, and reintroduce games as accessible for all audiences" (2014). Casual games therefore expand the notion of gamer identity because they are played by people other than the traditionally male hardcore gamer. In these respects, concerns about casual games in gamer culture come from the way they have extended gaming to people who are not seen as the target audience of video games.

Since casual games are so popular, game designers have tried to develop games that appeal to broader audiences. Chiapello noted that "the casual game phenomenon is widely acknowledged in the game design profession" (2014): the word "phenomenon" implies that casual games have redefined the ways that game designers create games. Such games are often designed to be played on mobile devices and for short periods of time, which leads to casual games being considered trivial in comparison to their hardcore counterparts. Players can get quite deeply involved in casual games, however, Hajinejad et al. argued that "casual games are not only games fitting into the gaps of everyday life" (2011), and many gamers play such games in a serious, hardcore way. Such elements blur the line between whether a game or player can be called casual or hardcore, and these authors all highlighted slippage between definitions of casual and hardcore with regards to video games, especially in terms of audience and design, suggesting that distinctions between casual and hardcore are complicated. The definitions deploy across a variety of other areas related to games as well, and Paavilainen et al. summarized the situation nicely: "casual is not a simple list of properties of a game. The phenomenon is an answer to a specific transformation of game cultures, forming a set of design values that correspond to these changes" (2009). Since the words are frequently used in discussions of game design and game players, the implications of the terms in those areas should be considered.

While factors such as rhetoric, audience and design are all useful elements to consider when discussing the terms casual and 
hardcore with respect to games, I focus here on how these concepts relate to the pricing structure used in free-to-play games. Shaw claimed that "we should also look at the dominant meanings encoded in the texts [players] are playing" (2010, 11), which means that the implications of the economic models built into freeto-play games like Hearthstone should be analyzed. The free-toplay purchasing model specifically adds a monetary dimension to casual games that is important to consider. Therefore, I argue that free-to-play casual games are structured in a way that promotes hardcore gameplay - and by extension, hardcore spending encouraging players to spend more on the game in addition to improving their skills. Given the implications of terms like casual and hardcore for both game design and gamer identity, this structure is problematic, suggesting that those with more money to spend on a game can more easily become a hardcore player, or even that a monetary investment is required to become one.

\section{METHODOLOGY}

In this paper, I use Hearthstone as a case study to demonstrate the effects of the free-to-play monetization model, illustrating the ways that money impacts casual and hardcore game design and gamer identity in free-to-play games. This impact can be seen in the pricing structure of the game, the ways that spending more on the game makes it more fun, the connection between money and time commitments required to play the game, and finally in the ways that players engage with gameplay elements based on both time and monetary constraints. To demonstrate this impact, I analyze the pricing, reward and ranking structures of Hearthstone as well as the player community surrounding the game.

To analyze the rhetoric surrounding the terms casual and hardcore among academics, I look at discussions about the terms in the critical game studies community. I have drawn on sources primarily from 2009 - 2018; as Hearthstone was released in 2014, this time frame offers a snapshot of critical discourse surrounding 
the terms casual and hardcore during Hearthstone's development, release and rise in popularity. In many cases, the discourse around these terms suggests that they are problematic and potentially even meaningless; however, their continued usage in critical circles suggests that discussions around these terms are worth considering.

In addition to critical sources, I use player-created paratexts such as game guides, discussions about the game on fan forums, and gaming news coverage of the game to illustrate how these elements are portrayed in gaming culture. I draw on some critical discourse surrounding these concepts as well, especially in regards to players streaming content on sites like Twitch.TV, but my primary focus is on the way the terms casual and hardcore are used within the player community.

Finally, I look directly at the game itself, in addition to the ways in which the game's developers have reinforced a monetary connection between casual and hardcore in Hearthstone's design. For this analysis, I rely on quotes from the developers and my own experience as a player: I have played Hearthstone regularly since its open beta in January 2014, though whether I would be considered a casual or hardcore player is probably up for debate!

\section{FREE-TO-PLAY COLLECTIBLE CARD GAMES}

Many modern casual games use the "free-to-play" model: such games rely on "microtransactions" in which players purchase game-related content for a small fee. Blizzard Entertainment, one of the largest game developers in the industry, created a free-toplay game based on their popular World of Warcraft franchise called Hearthstone. The game has become one of the most popular games in the free-to-play genre: a press release by Blizzard in May 2017 noted that Hearthstone had more than 70 million registered accounts (Campbell, 2017). It is also one of the most profitable: publicly available data released by an analyst firm in 2017 suggest that the game earns more than 25 million dollars a month (Minotti, 
2017). Hearthstone is a collectible card game: as in many others, Hearthstone players construct decks out of cards to battle one another. While Hearthstone offers some introductory cards to players completely for free, most of the game's cards are obtained through randomized card packs that must be purchased with reallife money or in-game currency. Cards are sorted into rarity levels - “common,” "rare,” “epic,” and "legendary” (Blizzard, 2014) - that roughly correspond to increasing levels of power, though power levels between cards and rarities can vary wildly. Players have a general idea of what cards could potentially be in a pack, as well as their chances of obtaining higher rarity cards, but do not find out what cards are inside until the pack is virtually opened. These card packs are sorted into expansions that are periodically released over time.

The digital card pack format described above is similar to purchasing a physical pack of cards for a collectible card game like Magic: The Gathering or Pokémon; however, it is worth noting some key differences. Players cannot trade cards with one another in Hearthstone as they can in real life, which was a deliberate design choice made to avoid high prices for desirable cards: in an interview at Gamescom in 2013 before the release of the game, executive producer Hamilton Chu commented, "We decided to go away from that" with regards to card trading (Goldfarb, 2013). Instead, Hearthstone players can use their old or duplicate cards to create a certain amount of "arcane dust” (Blizzard, 2014) that can be used to craft any card the player chooses of a lesser rarity. It is also worth noting that Hearthstone cards are not static, as real-life cards are: Blizzard occasionally updates problem cards to reduce their power level and has created a special "Standard" (Blizzard, 2014) format that only allows players to use newer cards to alleviate concerns about how difficult it is for newer players to compete against longtime players with large card collections. While these updates are presented as ways to keep the game fresh, they can be problematic for players who take long breaks from the game, as their old cards could be changed or become unusable in many gameplay formats, requiring them to purchase new cards. 
As with many other free-to-play games, Hearthstone players can obtain in-game currency that they can use to purchase card packs. Obtaining such currency can be time consuming, however, so most free-to-play games offer an alternative: purchasing content outright with real-life money. This pricing structure adds an economic element to considerations of casual and hardcore in freeto-play games, as the usual view within the player community is that "casual" and "free-to-play" are nearly synonymous (Marrilaife, 2016). On the other hand, the free-to-play model makes these games appealing to so-called casual players when compared to their physical equivalents like Magic, because those players never have to purchase content and can spend as little or as much as desired. In practice, however, players who want to succeed at free-to-play games like Hearthstone will probably need to purchase cards, as in-game currency is acquired faster through winning games or completing challenges in the game, and a larger collection of cards allows players to build more successful decks that win more frequently. Therefore, games such as these are sometimes derisively referred to as "pay to win" (Secant, 2017), a term that comes up often in discussion threads about Hearthstone. The pricing structure ensures that players who purchase content win more often because they have access to better options than players who have not spent money on content. Unlike a traditional game that players purchase and then play indefinitely, free-toplay games instead encourage players to periodically purchase new content, constantly pressuring them to spend more and suggesting that those who do are more hardcore players.

By relying on a free-to-play pricing structure, games like Hearthstone add an economic dimension to gameplay. As noted above, the ways people spend money in such games help to define casual and hardcore players in the eyes of the community: casual players spend less money on the game, while hardcore players spend more. From this perspective, the line between casual and hardcore games can be similarly drawn based on how much the game incentivizes purchasing content with real-life money: in other words, the more hardcore a game is, the more likely it is to 
employ a "pay-to-win” philosophy by giving gameplay advantages to players that pay for content. Most digital collectible card games like Hearthstone have a variable position on this spectrum because players purchase randomized content. In theory, a lucky player might get the content he or she wants very quickly and will therefore not have to spend much money. In practice, there will almost always be some content that a player does not have unless he or she spends significant amounts of money or time on the game to acquire all of it, and the periodical release of new content adds more opportunities for spending on the game over time. Randomization helps to alleviate some of a game's pay-to-win problems. However, in Hearthstone, for example, a player who spends more on the game might have more cards, but is not necessarily guaranteed to have better cards, and cannot simply purchase or trade for specific powerful cards outright. That being said, many critics have pointed out that the game has become more expensive over time: Friedman wrote an article titled "Hearthstone Has Become a \$400 a Year Game” and analyzed how the game’s pricing structure has changed since it was released, estimating that current Hearthstone players would now likely need to spend much more in order to get most of the cards released during a given year (2017). His analysis was based on assuming that such players would want to have access to most of the game's cards in order to build competitive decks, and he pointed out that "you don't have to chase every card, but the game is still aimed at making sure you spend more money than you have in the past" (Friedman, 2017). While his assumption may only be true for players who want access to most of the game's cards, the game is structured in a way that always encourages players to spend more. Spending money on a free-to-play game therefore allows a player to be more competitive against others, pushing them to become a more hardcore player in the eyes of the community. In addition, spending money on the game gives the player access to more cards, which makes it more fun to play. 


\section{FREE-TO-PLAY GAMES AND FUN}

As noted above, access to all of Hearthstone's content is not required to play the game, offering players a way to play the game without a significant monetary investment. Access to more content makes a player's game experience better because it offers more variety, however, such that fundamental concepts such as fun are impacted by the pricing structure of free-to-play games. Koster argued that "when you're playing a game, you'll only play it until you master the pattern; once you've mastered it, the game becomes boring" (2004, 14-18). As noted earlier, randomness is also a key part of the free-to-play monetization model, a trait that also contributes to fun in casual games. Juul pointed out that most casual games are simple and do not take hundreds of hours to complete as hardcore games do, so they often have various methods to reduce player boredom. One such mechanism Juul described was replayability, a way that casual games stay entertaining despite their simplicity: he provided Solitaire as an example of such a game $(2010,78)$. Hearthstone shares this trait, but differs from Solitaire because players construct multiple different decks to play with, using cards available from their library. Therefore, deckbuilding is an important aspect of the game's fun: access to new and different cards keeps players from getting bored, which incentivizes spending more on the game to get access to more cards.

Hearthstone also differs from Solitaire because it is a multiplayer game, so a player's deckbuilding choices are not made in a vacuum: the player builds his or her deck knowing that it will be tested against opposing decks, and a player's fun is often linked to how successful that deck is. These decks, and the decks a player's opponent uses, make up the patterns of Hearthstone, and are referred to as the game's "metagame," a term coined by Richard Garfield in reference to a similar situation in Magic: The Gathering (Carter et al., 2012). While players are free to make a deck out of any cards in their collection, the cards in Hearthstone are not all equal in terms of power or usefulness. The Hearthstone 
player community analyzes the game's expansions for cards that are more powerful than others and constructs "decklists" out of them, which are usually shared online: for example, one site claims to list "all of the currently viable decks" for a particular class (L0rinda, 2018). If one requirement of being a hardcore player within the player community is having access to the cards necessary to make a viable deck, a casual player is someone with few cards who can only build a few of these decks. Such players will master the pattern of those decks quickly and will likely get bored of them, especially if the decks are hard to win with. On the other hand, so-called hardcore players with lots of cards instead tend to gravitate toward established deck types, creating a metagame that often has specific, recognizable decklists. There are players who have fun by creating their own decks, but if those decks win frequently, they are quickly adopted by hardcore players, so even off-beat decks end up becoming part of the metagame. The metagame therefore structures the fun of playing Hearthstone: it determines many of the player's deckbuilding and gameplay choices, especially if they want to be competitive in the game.

The factors described above mean that access to more cards makes Hearthstone more fun, since a player with more cards can build a larger variety of decks. As such, Hearthstone's pricing structure incentivizes purchasing more content in multiple ways: having more cards allows a player to have more fun playing, encourages the player to be competitive and makes the player more of a hardcore player. As mentioned earlier, Hearthstone is also periodically updated with new cards: these updates keep the game from getting boring by introducing new cards and therefore new patterns into the metagame. The updates also mean that a player must continuously purchase new content to have fun and be competitive against other players. These elements add an economic factor to Hearthstone's fun because the game becomes less boring when the player spends more money on it: over time, players might find themselves spending many times the amount they would normally spend on a video game. For the game's 
casual players, those factors also contribute to the player's time investment into the game, since acquiring in-game currency allows a player to purchase more cards, have more fun and be more competitive.

\section{CASUAL GAMES AND TIME INVESTMENT}

The free-to-play pricing model also impacts the amount of time that players spend on these games. Juul argued that another key component of casual games is their flexibility: "a casual game is sufficiently flexible to be played with a hardcore time commitment, but a hardcore game is too inflexible to be played with a casual time commitment” (Juul, 2010, 12). Therefore, one way of defining the differences between casual and hardcore games is through the game's time requirements: a casual game is structured so that it does not require much time to play, and allows a player flexibility with how he or she spends that time. Likewise, Shaw argued that "video game culture is also defined in terms of the amount of time people spend doing it" $(2010,9)$, pointing out that the image of the hardcore player is typically one who spends a great deal of time playing the game. These viewpoints suggest that a casual or hardcore game is often defined in terms of how little time it requires of its players, and that a casual or hardcore player can be defined by how much time he or she spends playing a game.

Free-to-play games like Hearthstone, however, challenge the notion of the distinction between the quick casual game and the time-consuming hardcore game, as well as the notions about the time investment required from players in such games. In Hearthstone, the game's “quest” (Blizzard, 2014) system offers objectives for the player to complete for an in-game currency reward: a quest might require a player to use a certain type of card or a certain class. This design might sound flexible because players can simply not complete these quests if they would rather not invest the time required to do so. However, since the pricing structure of free-to-play games implies that distinctions between 
casual and hardcore are partly based on how much money a player has spent on the game, Hearthstone encourages a significant time commitment from its casual players. Players are given one quest every 24 hours, and they have a "quest log" (Blizzard, 2014) that can store up to three quests at a time, but once this quest log is full, any new quests are lost. Quests can also be "rerolled" (Blizzard, 2014) once a day, which gives the chance of granting a different quest instead. Since the quests award varying amounts of in-game currency, a player usually wants to maximize the value of his or her quest rewards, and will typically try to reroll less-valuable quests in the hope of getting more rewarding ones. The structure of Hearthstone's quest system therefore means that players who would rather spend in-game currency than real life money on the game must invest significant amounts of time optimizing their management of the system. Since there are a maximum number of quests that can be stored, players are encouraged to complete at least one quest every day, and since some quests have better rewards than others, players are also encouraged to reroll a quest every day to maximize their rewards. The result is that Hearthstone pressures casual players to play the game and complete quests every day to get as much in-game currency as possible, while hardcore players simply spend real-life money on the game instead, avoiding the time commitment necessary to complete the game's daily quests. Therefore, in free-to-play games, a casual player's time is more committed than a hardcore player's, unless he or she spends money to avoid those time investments, making the player more hardcore in the process.

These daily quest models are popular in many free-to-play games, and optimizing in-game currency rewards is so important to players that there are numerous online guides that offer advice on how to maximize those rewards. One Hearthstone guide claims that "you will end up spending far less money in the long run if you understand how to properly manage your in-game resources" (Aleco, 2017), suggesting that a great deal of a casual Hearthstone player's time is spent trying to complete these quests to save themselves money. Because casual free-to-play games use a 
reward structure that provides players with currency, time investment is directly tied to the game's pricing structure. Since hardcore players have spent a great deal of money on the game, such players have little incentive to complete these quests compared to casual players, as they have no need for in-game currency. In the case of Hearthstone, hardcore players are more likely to have spent money on the game to get the cards they want, and therefore are less likely to need the in-game currency offered by the game's daily quests. Hearthstone's hardcore players often ignore the game's quest system entirely, as they have no reason to spend their time completing those quests. The game's casual players, on the other hand, have a strong incentive to try to maximize the currency they gain from quests: since they spend less real-life money on the game, quests provide the in-game currency needed to get access to more cards.

While hardcore players likely play more than casual players because they are more invested in gaming as a hobby, free-toplay games like Hearthstone challenge Juul's notion of flexibility in casual games because the time hardcore players spend in the game is less committed. In Hearthstone, casual players must play daily to keep up with their quests, while hardcore players do not have such concerns. Both types of players might invest a great deal of time in the game, but hardcore players have more freedom with how they spend their in-game time and focus more on the game's ranked play system, where winning offers minimal in-game currency rewards, but confers hardcore status in Hearthstone's gaming community. Since the game's ranked play mode is skill-intensive, many players often avoid that game mode; in fact, the game reinforces the distinction between casual and hardcore by offering an unranked mode that is referred to as "casual play" (Blizzard, 2014). The name of the game modes suggests that the ranked play mode is considered the game's more hardcore play mode by the game's designers themselves, and influences the community's play practices. One of the game's main developers, Ben Brode, described the game mode this way: “casual mode, ideally, is just low stakes. I don't have to worry 
about losing anything or having loss aversion... It isn't hardcore competition where I need to be 100 percent focused and available” (Wilson, 2017). That the game's developers themselves make this distinction is problematic because it reinforces a connection between perceived player status and how much they spend on the game: ranked players are usually seen as more skilled and therefore more hardcore, but they are also likely to have spent money to get the cards necessary to build a competitive deck, while casual players have not. These factors mean that the economic elements that impact casual and hardcore players in freeto-play games are not only influenced by how much time a player spends playing: it is also important to consider spending in relation to how players spend their time in the game. In free-to-play games, how much a player spends on the game determines how he or she plays it, which likewise determines the player's status as casual or hardcore. In Hearthstone, such differences can be observed through play practices in the game's ranked play mode and in its competitive tournament scene.

\section{CASUAL AND HARDCORE PLAY PRACTICES}

Differences in casual and hardcore play practices can be seen in the way players engage with Hearthstone's daily quests and advancement systems when compared to other games, as well as in how those systems are structured. These differences imply that it is important to consider how players spend their time in free-to-play games in addition to considering how much time players spend playing them or how much time is required to play the game, since play practices themselves are influenced by the game's pricing structure. Shaw argued that "gaming can be, and has been, studied in terms of play practices” (2010, 9): how players play games is an important paradigm for studying them. In many games, how a player spends his or her time determines whether that player is casual or hardcore in the eyes of the community, and in free-toplay games, the pricing structure and game design both contribute to casual or hardcore play practices. 
The ways a video game structures rewards and advancement are often the main factors in determining a player's play practices: for example, in games like World of Warcraft, daily quests are timeconsuming endeavors that are usually undertaken by the game's most hardcore players. In hardcore games, daily quests offer a specialized set of objectives designed for players who already spend a great deal of time playing and are aimed at creating a continuous path of advancement that provides a reward structure for hardcore players. In free-to-play games like Hearthstone, however, daily quests are not part of the game's advancement system, since the only reward for completing them is in-game currency; these quests are instead aimed at the game's casual players in the hopes of getting them to play more often. In fact, since the amount of in-game currency that a player can obtain is capped and there is a limited amount of content to spend it on, Hearthstone's most hardcore players ignore these daily quests entirely, since those players have spent enough money on the game to have all the content they want.

Rather than being tied to daily objectives and reward systems, a player's casual or hardcore status in Hearthstone is instead tied to the player's performance in spaces such as the game's ranked play mode. While the game's ranked play mode offers rewards for achieving certain ranks, these rewards are given out on a monthly basis and are quite minimal in comparison to the rewards given by the quest system. The game mode is instead intended for hardcore players who are interested in testing their skills against others, and a high ranking is seen as a status symbol in the Hearthstone community: Blizzard often releases news updates detailing the top-ranked players each month (Blizzard Entertainment, 2018). In competitive free-to-play games like Hearthstone, advancement is measured differently than in games like World of Warcraft: hardcore Hearthstone players focus on the game's rank-based advancement system, as well as specialized organized tournaments with specific rules that are held outside of the game's ranked play structure. 
Hearthstone's ranked play mode consists of 50 in-game ranks that a player advances through as he or she plays each month: winning games increases a player's rank, while losing decreases it. A player's rank is reduced at the beginning of each month, meaning that consistently achieving a high ranking in the game's ranked play mode requires the kind of regular, hardcore time investment that Juul discussed. Once a player has reached rank 1, the player can advance to "legend" (Blizzard, 2014) rank, a specialized ranking system for the best players that provides a direct numerical ranking of the player's status against other legend players within their region. Consistently reaching legend rank is one of the main markers of a hardcore Hearthstone player within the community. While gameplay skill is usually seen as the most significant determining factor of a player's ability to reach legend rank, there is a monetary factor to reaching legend rank as well: a player must have access to enough cards to build an effective deck that can win consistently, something that is very difficult to do with a limited set of cards. There is also a time investment element required to achieve the legend rank: while Blizzard has updated the ranked mode over the years, fans typically estimate that players will need to win hundreds of games to reach legend rank each month (Berry, 2017). These factors suggest that gameplay skill is tied to money and time investment in these games: while a player might be good at the game to achieve the legend rank, he or she also needs to have the cards necessary to build decks that can reach that rank.

Consistently achieving legend status in the game's ranked play mode also often gets players invited to special invitation-only Hearthstone tournaments, the largest of which are sponsored by Blizzard themselves. These tournaments form the backbone of the game's “competitive scene," and each year, the best Hearthstone players compete at a worldwide tournament to crown a world champion. As in many other collectible card games, Hearthstone tournaments are typically seen as the pinnacle of competitive play: there are professional Hearthstone players who make a living by competing in these tournaments and winning prize money, and fan 
websites that rank the players by earnings and tournament success (GosuGamers, 2018). Professional players often also stream their in-game playtime through services such as Twitch T.V., which authors such as Johnson and Woodcock have described as a rapidly growing career path for gamers (2017). These factors combine to create an interesting reversal of the game's economic structure: the most hardcore of Hearthstone's players can achieve a kind of celebrity status in the community, and are even able to make a living from their Twitch streams and tournament winnings. While this description only applies to a small percentage of Hearthstone players, it also represents perhaps the most extreme example of the economic implications of free-to-play game structures: the game's most hardcore and skilled players have invested so much time and money into the game that they can see a return on their money if they make an effort to monetize their playtime.

\section{CONCLUSION}

While there are many elements to consider about the rhetoric surrounding casual and hardcore with regards to players and games, the monetary factor is particularly problematic in free-toplay games, because it is so closely linked to status and identity: a player needs enough content to be competitive if he or she wants to be hardcore, and spending money is the fastest and easiest way to get that content, suggesting that players should do that first and improve their gameplay skills afterward. These factors therefore complicate notions of casual and hardcore in free-to-play games. Complicating the issue further is that time, economics and status are all linked in free-to-play games: spending money on the game allows players to spend their time on hardcore elements of the game, such as its ranking system or competitive tournaments, while not doing so means players will instead need to spend time gaining in-game currency in order to become more hardcore. This is problematic because gameplay and players are often separated by the terms, casual and hardcore, in the ways they are used by both the gaming community and game developers. In free-to-play 
games, access to hardcore elements is restricted by the player's ability to pay for content, suggesting that those who do not have the money to spend are casual players and that they cannot become hardcore players until they do so. Considering that casual games and players are often portrayed negatively, this linkage between time, money and status in free-to-play games like Hearthstone is especially concerning.

The economic aspects of free-to-play casual games also open new avenues of analysis for the future. Specifically, analyses of the capitalistic elements of this monetization method would be particularly valuable, and, as noted earlier, some gaming journalists have already highlighted the exploitative nature of this pricing structure. The influence of the free-to-play pricing structure on non-digital games has also been noted (Maisenhölder, 2018), suggesting that it may impact all kinds of games as well. Since this method of pricing has become particularly popular with casual mobile games, which have greatly expanded the gaming audience in general, it is unlikely to go away any time soon, and further critical analysis of it could therefore be quite useful. While I have focused primarily here on structural analysis of one of the most popular free-to-play games in the industry, the implications of this pricing model, especially in games that are more aggressively "pay-to-win” than Hearthstone, might be analyzed from Marxist or cultural perspectives that could draw out many other problematic elements inherent in such an aggressively capitalistic monetization structure. One troubling aspect of such games is that players with more disposable income and time are more likely to succeed than players who do not. This element of free-to-play-games suggests that the constant pressure to spend money and become more hardcore, as highlighted here, reflects some of the larger problematic issues in capitalistic societies that link social status to money, and I believe that further work in this area could be particularly useful.

It is not surprising that free-to-play games like Hearthstone complicate terms like casual and hardcore, however, since the 
game is part of a genre that has exploded in popularity and has generated a great deal of discussion about casual game designers and casual gamers. While that model has also been criticized - as noted earlier, a common negative descriptive term for these games is that they are pay-to-win instead of free-to-play - it has also come to dominate the mobile game industry. These factors suggest that one useful way to consider a free-to-play game is to examine the implications of its economic structure and reward systems, and that players can be evaluated based on how much money they spend on a game, as well as how they spend their time playing it.

\section{BIBLIOGRAPHY}

Aleco. "Beginner's guide to spending gold.” Icy Veins, 2017. https://www.icy-veins.com/hearthstone/beginners-guide-tospending-gold

Berry. "How many games needed to reach legend?" Hearthstone Forums, 2017. https://us.battle.net/forums/en/hearthstone/topic/ 20754497783

Blizzard Entertainment. Hearthstone [Online Game]. Blizzard Entertainment, 2018.

Blizzard Entertainment. "Top Hearthstone Players - January 2018.” Blizzard.com, 2018. https://news.blizzard.com/en-us/ hearthstone/21513836/top-hearthstone-players-january-2018

Campbell, M. “Hearthstone passes 70 million players.” IGN, 2017. http://www.ign.com/articles/2017/05/01/hearthstonepasses-70-million-players

Carter, M., M. Gibbs, and M. Harrop. "Metagames, Paragames, and Orthogames: A New Vocabulary.” In FDG '12, 2015. http://dl.acm.org/citation.cfm?id=2282346 
Chess, S. "Going with the Flo: Diner Dash and Feminism." Feminist Media Studies vol. 12, no. 1(2014): 83-99.

Chiapello, L. "Formalizing Casual Games: A Study Based on Game Designer's Professional Knowledge.” In DiGRA '13 Proceedings of the 2013 DiGRA International Conference: DeFragging Game Studies. DiGRA, Atlanta, GA, USA, 2014. http://www.digra.org/wp-content/uploads/digital-library/ paper_168.pdf

Eklund, L. "Who are the Casual Gamers? Gender Tropes and Tokenism in Game Culture.” In Social, Casual, and Mobile Games: The Changing Games Landscape, edited by Tama Leaver and Michele Willson, pp. 15-30. New York, NY, USA, Bloomsbury Academic, 2016.

Freidman, D. "Hearthstone Has Become a $\$ 400$ a year game." Polygon, 2017. https://www.polygon.com/2017/12/12/16763594/ hearthstone-expensive-expansions-cost

Goldfarb, A. "GamesCom: The Origin and Future of Blizzard's Hearthstone.” IGN, 2013. http://www.ign.com/articles/2013/08/ 24/gamescom-the-origin-and-future-of-blizzards-hearthstone

Hajinejad, N., I. Sheptykin, B. Grüter, A. Worpenberg, A. Lochwitz, D. Oswald, and H. Vatterrott. "Casual Mobile Gameplay - On Integrated Practices of Research, Design, and Play." In DiGRA '11 - Proceedings of the 2011 DiGRA International Conference: Think Design Play. DiGRA, Utretcht, Netherlands, 2011. http://www.digra.org/wp-content/uploads/ digital-library/11301.00075.pdf

GosuGamers. "Hearthstone Rankings Database." GosuHearthstone, 2018. https://www.gosugamers.net/hearthstone/ rankings

Juul, J. A Casual Revolution: Reinventing Video Games and Their Players. Cambridge, MA, USA: The MIT Press, 2010. 
Johnson, M. and J. Woodcock. “'It's Like the Gold Rush': The Lives and Careers of Professional Video Game Streamers on Twich.TV." Information, Communication, and Society, 2017. https://www.tandfonline.com/doi/full/10.1080/

1369118X.2017.1386229

Koster, R. A Theory of Fun for Game Design. Scottsdale, AZ, USA: Paraglyph Press, 2004.

Marrilaife. "F2P Casual Player: What Decks to Focus on?” MMOChampion, 2016, https://www.mmo-champion.com/threads/ 1949068-F2P-casual-player-what-decks-to-focus-on

L0Rinda. "Hunter Decks - Hearthstone.” Icy Veins, 2018. https://www.icy-veins.com/hearthstone/hunter-decks

Maisenhölder, P. "Why should I play to win if I can pay to win?: Economic inequality and its influence on the experience of nondigital games." Well Played vol. 7, no. 1(2018): 60-83.

Minotti, M. "SuperData: Hearthstone Trumps All Comers in Card Market That Will Hit 1.4 Billion in 2017." Venturebeat, 2017. https://venturebeat.com/2017/01/28/superdata-hearthstonetrumps-all-comers-in-card-market-that-will-hit-1-4-billionin-2017/

Paavilainen J, A. Kultima, J. Kuittine, F. Mäyrä, H. Saarenpää, and J. Niemelä. "GameSpace: Methods for Design and Evaluation for Casual Mobile Multiplayer Games.” Tampere: University of Tampere, Department of information studies and interactive media, 2009. (Research of interactive media 1).

Paul, C.A. "Optimizing Play: How Theorycraft Changes Gameplay and Design” Game Studies vol. 11, no. 2 (2011). http://gamestudies.org/1102/articles/paul

Poels, Y, J. H. Annema, M. Verstraete, B. Zaman, and D. De Grooff. 2011. "Are you a Gamer? A Qualitative Study Concerning 
the Parameters Used to Categorize Casual and Hardcore Gamers.” IADIS International Journal on WWW/Internet, 10, (1), 1 - 16.

Secant. "This game is $100 \%$ pay to win." Hearthstone Forums, 2017 https://us.battle.net/forums/en/hearthstone/topic/ 20758496548?page $=1$

Soderman, B. "Killing Time in Diner Dash: Representation, Gender, and Casual Games.” In DiGRA '09 - Proceedings of the 2009 DiGRA International Conference: Breaking New Ground: Innovation in Games, Play, Practice, and Theory. DiGRA, London, United Kingdom, 2009. http://www.digra.org/digitallibrary/publications/killing-time-in-diner-dash-representationgender-and-casual-games-abstract/

Shaw, A. "What is Video Game Culture? Cultural Studies and Game Studies.” Games and Culture vol. 5, no. 3(2010): 403 - 424.

Wilson, J. "Hearthstone Boss Ben Brode gives me new insight on what 'casual' means.” Venturebeat, 2017, https://venturebeat.com/ 2017/10/09/hearthstone-boss-ben-brode-gives-me-new-insighton-what-casual-means/ 